D.T. Wickramasinghe, L. Ferrario, and G.V. Bicknell, eds.

\title{
HST Spatially Resolved Spectra Of Accretion Discs In CVs
}

\author{
Raymundo Baptista \\ School of Phys. \& Astronomy, University of St. Andrews, Scotland \\ Depto. Física, Universidade Federal de Santa Catarina, Brazil
}

\begin{abstract}
Time-resolved eclipse spectroscopy of the novalike variable UX UMa obtained with the HST/FOS is analized with eclipse mapping techniques to produce spatially resolved spectra of its accretion disk and gas stream as a function of distance from disk center. The results reveal a noticeable asymmetry in the disk emission, with the disk side closest to the secondary star showing pronounced absorption bands in the UV and a Balmer jump in absorption. The spectrum of the infalling gas stream is noticeably different from the disk spectrum at the same radius suggesting that gas overflows through the impact point at disk rim and continues along the stream trajectory, producing distinct emission down to $0.1 R_{\mathrm{L} 1}$.
\end{abstract}

\section{Introduction}

Cataclysmic Variables (CVs) provide an excellent opportunity for observational studies of accretion disks with the aid of maximum-entropy eclipse mapping techniques. These techniques have advanced to the stage of delivering spatiallyresolved spectra of accretion disks (Rutten et al. 1994, Baptista et al. 1995), offering a vast improvement in the observational constraints on disk atmosphere models. This paper reports on the spectral mapping of the novalike variable UX UMa based on observations made with the Faint Object Spectrograph (FOS) on the Hubble Space Telescope (HST).

\section{Data analysis}

Time-resolved spectroscopy centered on eclipses of UX UMa was obtained with HST/FOS on 1994 August (G160L, $1100-2500 \AA$ ) and 1994 November (PRISM, $1600-8500 \AA)$ at a time resolution of $5.3 \mathrm{~s}$, in a total of 4 data sets and 3122 spectra. Data reduction procedures included background subtraction, flat-field correction, and wavelength and flux calibration. A brief description of the data set can be found in Baptista et al. (1996).

The calibrated spectra were divided into 59 (G160L) and 127 (PRISM) passbands and light curves were constructed for each one. Maximum-entropy eclipse mapping techniques (Horne 1985; Baptista \& Steiner 1993) were used to solve for a map of the disk brightness distribution and for the flux of an additional uneclipsed component in each band. The eclipse maps were combined to produce 


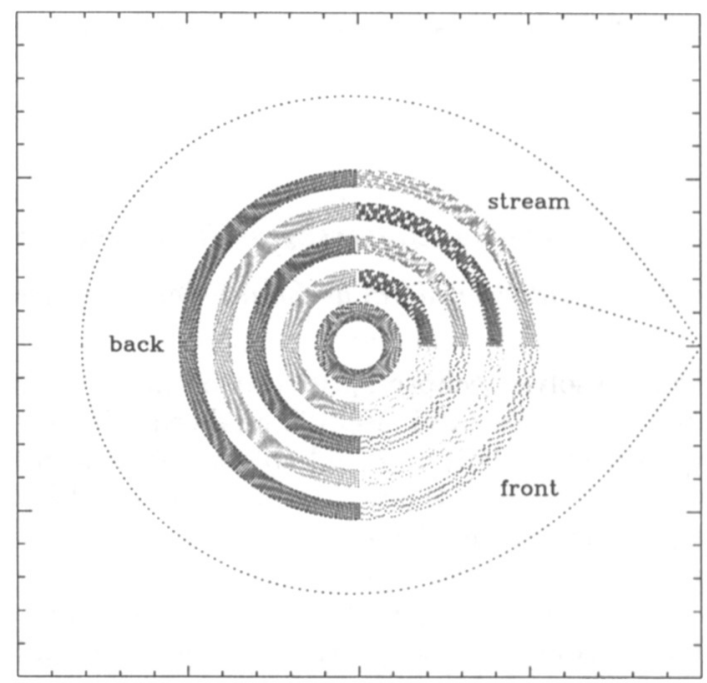

Figure 1. Annular regions used to extract spatially resolved spectra. Each ring has a width of $0.05 R_{\mathrm{L} 1}$, where $R_{\mathrm{L} 1}$ is the distance from disk center to the inner Lagrangian point. Dotted lines show the primary Roche lobe and the theoretical gas stream trajectory. The secondary star is to the right of the panel and the stars rotate counter-clockwise.

spatially resolved spectra for a set of concentric annuli on three major azimuthal regions: the back side of the disk, the front side of the disk, and the gas stream region (Fig. 1).

\section{Results}

Spatially resolved spectra of the back side region show that the inner disk in the UV is characterized by a blue continuum filled with absorption lines and bands which cross over to emission with increasing disk radius, in accordance with the findings of Rutten et al. (1994) in the optical.

The comparison of the front and back side spectra at the same radius reveals a significant asymmetry in the disk emission at UV wavelengths, with the front side of the disk exhibiting pronounced absorption bands and a Balmer jump in absorption (Fig.2). This effect becomes more pronounced for increasing disk radius and can also be noted in the back side spectrum at large radii. These remarks suggest the existence of an absorbing ring of cold gas in the outer disk, probably reminiscent of the "Fe II curtain" as observed in OY Car (Horne et al. 1994).

Figure 3 shows the difference between the spectrum of the stream and that of the front side of the disk as a function of disk radius. The spectrum of the gas stream is noticeably different from the disk spectrum at the same radius for a range of radii. The spectrum of the difference becomes progressively bluer with decreasing disk radius. This suggest that gas overflows through the impact 


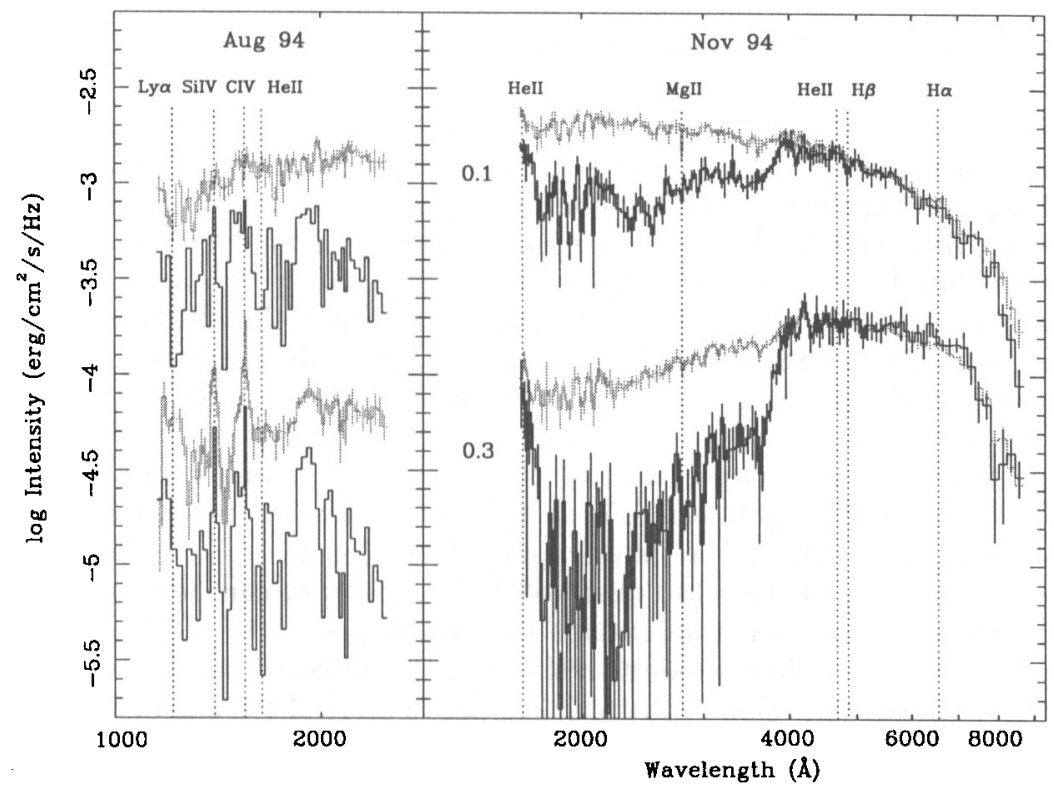

Figure 2. Comparison of spatially resolved spectra of the back side (light gray) and the front side of the disk (black) at two different annuli (labels in units of $R_{\mathrm{Ll}}$ ). Error bars were computed from Monte Carlo simulations with the light curves.

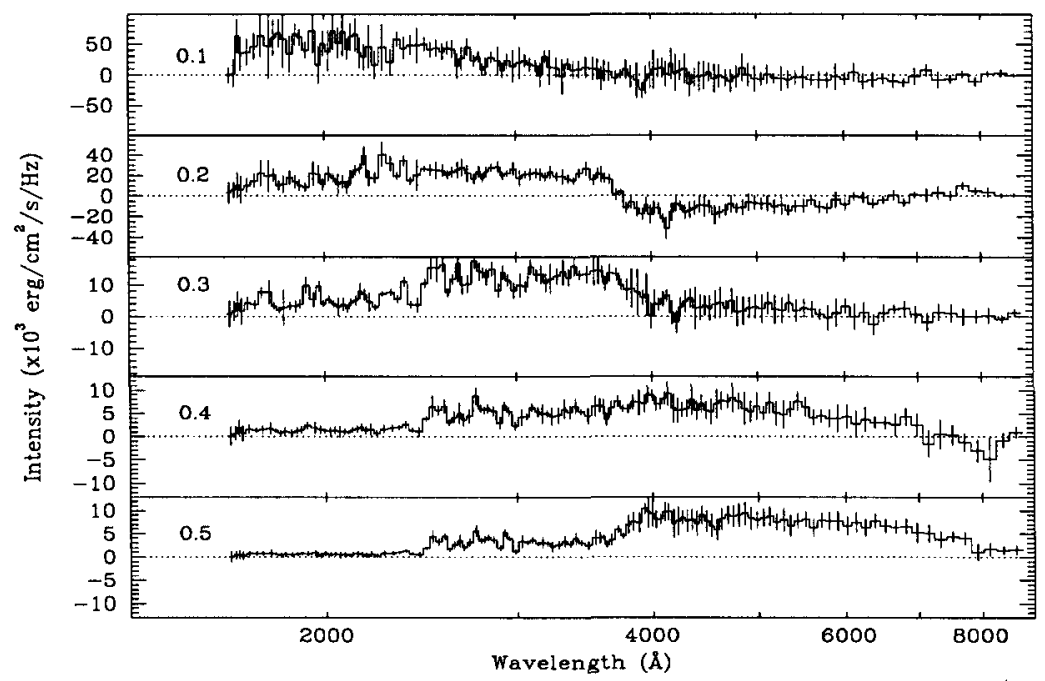

Figure 3. The difference between the gas stream and the front side spectra for a set of annuli (labels in units of $R_{\mathrm{L} 1}$ ). 
point at disk rim and continues along the stream trajectory, producing distinct emission down to $0.1 R_{\mathrm{L} 1}$.

The comparison of the 1994 Aug and Nov eclipse maps shows a significant $(\simeq 50 \%)$ increase in brightness in the inner disk regions at the later epoch. The radial temperature profiles of the continuum maps are well described by a steady-state disk model. There is marginal evidence of an increase in the mass accretion rate from $A u g$ to $\operatorname{Nov}\left(\dot{\mathrm{M}}=10^{-8.3 \pm 0.1} \mapsto 10^{-8.1 \pm 0.1} \mathrm{M}_{\odot} \mathrm{yr}^{-1}\right)$, in accordance with the observed increase in brightness. Since the UX UMa disk seems to be in a high-viscosity state in both cases, this result suggests that the mass transfer rate of UX UMa varies by a substantial amount $(\simeq 50 \%)$ on time scales of a few months.

The spectrum of the uneclipsed light shows prominent emission lines (Ly $\alpha$, Si IV, CIV, He II, MgII), significant UV continuum emission (up to $\simeq 20 \%$ of the total light at $3600 \AA$ ), and a Balmer jump in emission. One interesting question which arises is whether the observed UV continuum can be explained in terms of disk wind emission from outside the orbital plane.

By fitting disk atmosphere models to the spatially resolved spectra we will be able to derive the distribution of the physical parameters of the accretion disk - its temperature, surface density, Mach number, optical depth, and vertical temperature gradient.

Acknowledgments. This work was partially supported by NASA grant GO-5488 from the STScI (which is operated by AURA under NASA contract NAS 5-26555) and by CNPq/Brazil research grant no. 300 354/96-7.

\section{References}

Baptista, R.; Horne, K.; Long, K.; Hubeny, I.; Mauche, C.W.; Rutten, R.G.M. \& Wade, R.A. 1996, in Proceedings of IAU Colloquium 158: Cataclysmic Variables and Related Objects, ed. A. Evans \& J. H. Wood, (Dordrecht: Kluwer), in press

Baptista, R.; Horne, K.; Hilditch, R.; Mason, K.O. \& Drew J.E. 1995, ApJ, 448, 395

Baptista, R. \& Steiner, J.E. 1993, A\&A, 277, 331

Horne, K. 1985, MNRAS, 213, 129

Horne, K., Marsh, T.R.; Cheng F.-H.; Hubeny, I. \& Lantz, T. 1994, ApJ, 426, 294

Rutten, R.G.M.; Dhillon, V.S.; Horne, K. \& Kuulkers, E. 1994, A\&A, 283, 441

\section{Discussion}

J. Smak: Two effects could be responsible for the back-side/front-side difference: (1) The self-obscuration of the front-side by the disk rim making the front-side cooler and fainter, (2) The circumdisk absorption, concentrated in front of the hot spot, which - due to the relatively low inclination - also affects primarily the front-side. This effect should be particularly strong in the UV, which seems to be indeed the case. 